\title{
STIMULATED EMISSION in Zn(Se,S) SINGLE CRYSTALS
}

L. KowalczyK, A. Mycielski, A. SzaDkowski

Institute of Physics, Polish Academy of Sciences

Al. Lotników 32/46, 02-668 Warszawa, Poland

C. Testelin, C. Rigaux and M. Menant

Groupe de Physique des Solides, Universités Paris VI et Paris VII

Tour 23, 2 place Jussieu, 75251 Paris Cedex 05, France

Two different types of optically pumped stimulated emission were observed at $1.8 \mathrm{~K}$ in the high quality $\mathrm{Zn}(\mathrm{Se}, \mathrm{S})$ crystals grown by low temperature physical vapour transport. One type, occurring at moderate excitation levels, is ascribed to the recombination of excitons localized because of chemical disorder in the two-anion mixed crystal. The other type, occurring at high excitation levels, is related to the inelastic exciton-exciton scattering.

PACS numbers: $78.45 .+\mathrm{h}, 78.55 . \mathrm{Et}, 81.10 . \mathrm{Bk}$

The high quality $\mathrm{Zn}(\mathrm{Se}, \mathrm{S})$ crystals were grown by low temperature physical vapour transport technique. The photoluminescence was investigated at $1.8 \mathrm{~K}$. The samples were immersed in pumped liquid helium. Two different ways of optical excitation were used: a continuous beam from a $7 \mathrm{~mW} \mathrm{He}-\mathrm{Cd}$ laser $(3.8 \mathrm{eV})$ or pulses $(5 \mathrm{~ns}, 10 \mathrm{~Hz})$ from a frequency tripled Nd:YAG laser $(3.49 \mathrm{eV})$.

$\mathrm{He}-\mathrm{Cd}$ laser was used to obtain the photoluminescence spectra for the low excitation levels. Opposite to the rich luminescence spectra, which we obtained for $\mathrm{ZnSe}$ and for the two-cation mixed crystals $(\mathrm{Zn}, \mathrm{Mg}) \mathrm{Se}$, the near-edge spectra of the two-anion crystals were usually limited to one or two lines in the vicinity of the free exciton (FE) energy.

As the energy gap and the free exciton energy depend on the composition of a ternary crystal, we measured the reflectivity spectrum in order to determine the position of the free exciton structure. Then, the photoluminescence lines were reliably located with respect to the middle of the $\mathrm{FE}$ structure in reflection.

A $\mathrm{ZnSe}_{0.88} \mathrm{~S}_{0.12}$ crystal was chosen to detailed studies. The low-excitation photoluminescence spectra of the samples from this crystal contained mainly one strong line in the near band-edge region. A second line, 5-6 meV below $\mathrm{FE}$, was more or less pronounced in various samples. The second line can be easily identified as belonging to the donor-bound exciton (see for example [1]). The concentration 

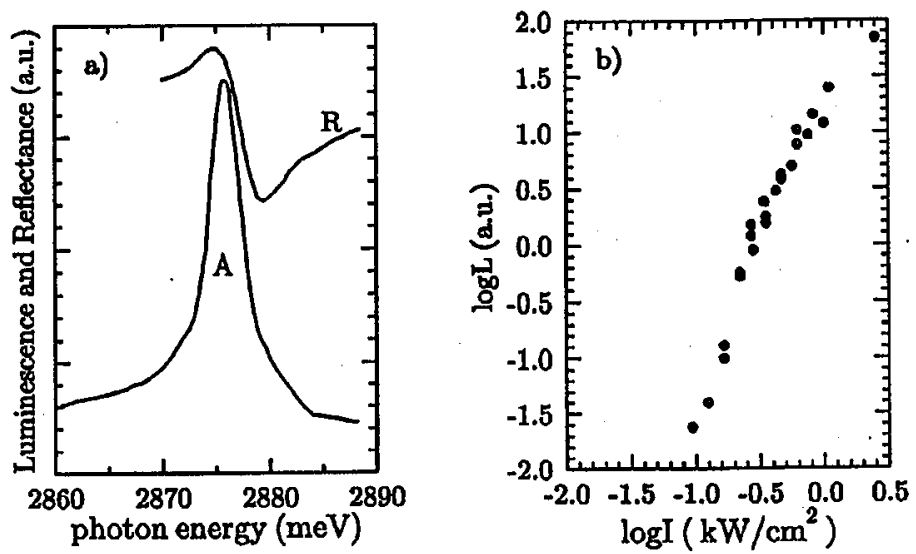

Fig. 1. (a) Near-band-edge photoluminescence spectrum (line A) for low (a few $\mathrm{mW} / \mathrm{cm}^{2}$ ) continuous excitation, compared with the reflection spectrum (R) in the $\mathrm{ZnSe}_{0.88} \mathrm{~S}_{0.12}$ sample at $1.8 \mathrm{~K}$. (b) Intensity $L$ of the line A versus excitation power $I$; pulsed excitation.

of the residual donor impurities may vary inside the crystal. We will focus our attention on the first line. The dominating role of this line seems to be an inherent property of the mixed-anion crystals.

Figure 1 presents the near-band-edge luminescence and reflectivity spectra of the $\mathrm{ZnSe}_{0.88} \mathrm{~S}_{0.12}$ sample. Both the luminescence and reflection were measured in the same experimental setup for the same small area of the sample surface. The luminescence spectrum consists of just one line observed about $1.5 \mathrm{meV}$ below the middle of FE structure, and it certainly does not belong to a donor-bound exciton. Having only the position we cannot infer the origin of this line (we will call this line " $A$ "). Such line for $\mathrm{Zn}(\mathrm{Se}, \mathrm{S})$ was observed by Huang et al. [2]. They ascribed it simply to FE. We are going to show stimulated emission related to the line $\mathrm{A}$. As the free excitons are bosons, it is difficult to imagine the stimulated emission related to FE. This is the reason why we doubt in the assignment (as in Ref. [2]) of the line $\mathrm{A}$ to $\mathrm{FE}$. We ascribe the line $\mathrm{A}$ to the recombination of excitons localized because of chemical disorder, which is always present in the ternary crystals.

The photoluminescence spectra and the dependence of the luminescence intensity on the pumping power were studied in the wide range of excitation levels. The intensity $L$ of the line A versus excitation intensity $I$ in the region $0.1-3.0 \mathrm{~kW} / \mathrm{cm}^{2}$ is presented in Fig. 1b. An overlinear dependence of $L$ on $I$ is clearly seen. If this dependence is approximated by the expression: $L=C I^{k}$, the value of $k$ exceeds 2.8. Such a high value of $k$ gives strong evidence of the stimulated emission.

The stimulated emission related to the excitons localized because of chemical disorder has not been reported yet for the bulk II-VI mixed crystals, but it is known (see e.g. [3]) that the excitons localized because of a certain disorder in quantum-well structures (width fluctuations, stress inhomogeneity, etc.) take 


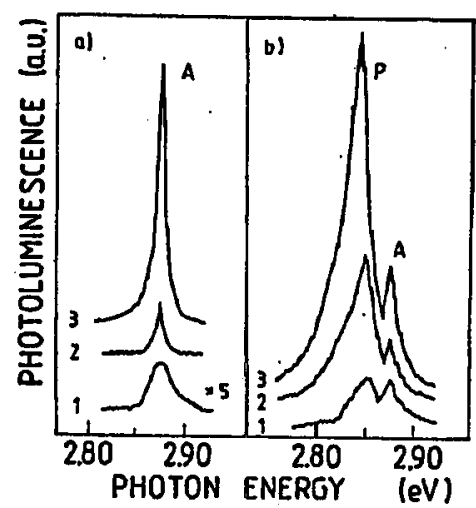

Fig. 2. Near-band-edge photoluminescence spectra for various powers of pulsed excitation in the $\mathrm{ZnSe} \mathrm{e}_{88} \mathrm{~S}_{0.12}$ sample at $1.8 \mathrm{~K}$. (a) The powers used for spectra 1,2 and 3 were $0.6,2$ and $3.4 \mathrm{~kW} / \mathrm{cm}^{2}$, respectively. (b) The powers used for spectra 1,2 and 3 were 20,52 and $69 \mathrm{~kW} / \mathrm{cm}^{2}$, respectively.

part in stimulated emission processes. As we wrote above, the line $\mathrm{A}$ seems to be characteristic of the two-anion systems. We explain the different behaviour of the two-cation and the two-anion systems by different effectiveness of the localization of the hole (this effectiveness is higher in the two-anion crystal, where the band-offset occurs mainly in the valence bands).

As it can be seen in Fig. $2 a$, the line $A$ is the only feature of the luminescence spectrum for the excitation powers below about $10 \mathrm{~kW} / \mathrm{cm}^{2}$. The luminescence spectra obtained for the highest excitation levels are presented in Fig. 2b. Now, the peak $A$ is accompanied by a new peak $P$, which increases rapidly with increasing excitation and soon becomes dominant. The peak $P$ emerges about 28 meV below A and goes down in energy ("red shift") for increasing excitation. The positions of both peaks as functions of the excitation intensity are presented in Fig. 3a. The heights of the peaks versus the excitation intensity are shown in Fig. $3 \mathrm{~b}$. Different behaviour of the peaks is evident. Superlinearity of the $\log -\log$ plot for the peak $\mathrm{P}$ starts at about $100 \mathrm{~kW} / \mathrm{cm}^{2}$ and indicates the onset of the second kind of stimulated emission at high excitation densities.

Following [4] and [5], where the inelastic exciton-exciton scattering was proposed as a mechanism of stimulated emission in $\mathrm{ZnSe}$, we explain the nature of the peak $\mathrm{P}$ and its red shift by this type of interaction of two excitons. The first exciton dissociates into an electron-hole pair, taking at least $E_{\mathrm{b}}$ (binding energy of the exciton) from the annihilation energy of the second exciton. The scattering process results in a photon leaving the crystal (peak P) with the energy lowered with respect to the annihilation energy of a single exciton by at least $E_{\mathrm{b}}$. This lowering of the photon energy, increases for high excitation densities, when the bands become highly populated and the dissociation of an exciton into an electron-hole pair requires additional energy to transfer both particles deeper into the bands, beyond the quasi-Fermi levels. This band-filling effect causes the red shift (presented in Fig. 3a) of the peak $\mathrm{P}$ at very high excitation densities. 

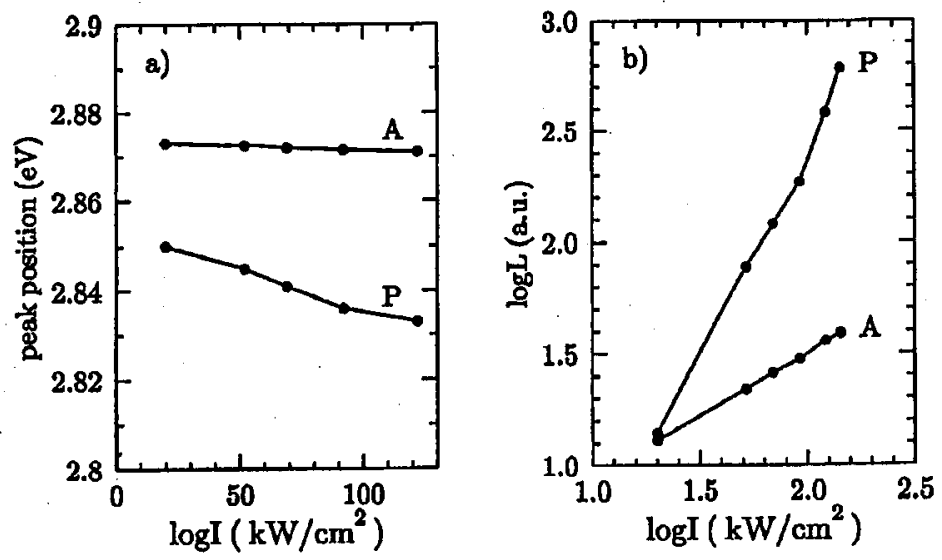

Fig. 3. Positions (a) and intensities (b) of the photoluminescence lines $A$ and $P$ from Fig. 2 versus excitation power $I$ in the region of high excitation (as in Fig. 2b).

Concluding: (1) We observe a luminescence line very close to the free exciton structure in reflection and we defy the interpretation of this line as free exciton because we observe the stimulated emission of this line. (2) We also observe the stimulated emission of a line more than $23 \mathrm{meV}$ below the FE and we ascribe this line to inelastic exciton-exciton scattering.

\section{References}

[1] Xi-Min Huang, K. Igaki, J. Cryst. Growth 78, 24 (1986).

[2] Xi-Min Huang, S. Satoh, K. Mochizuki, K. Igaki, Jpn. J. Appl. Phys. 22, 674 (1983).

[3] J. Ding, H. Jeon, T. Ishihara, M. Hagerott, A.V. Nurmikko, H. Luo, N. Samarth, J. Furdyna, Phys. Rev. Lett. 69, 1707 (1992).

[4] P.R. Newbury, K. Shahzad, D.A. Cammack, Appl. Phys. Lett. 58, 1065 (1991).

[5] X.H. Yang, J.J. Song, E. Cantwell, J. Aldridge, Phys. Status Solidi A 127, K79 (1991). 